\section{LETTERS TO THE EDITORS}

The Editors do not hold themselves responsible for opinions expressed by their correspondents. No notice is taken of anonymous conmunications

\section{Is there an Fther?}

IN Nature of November 24, p. 906, Dirac draws an interesting conclusion from his new electrodynamics; namely, the necessity of a revival of the rether concept. I have some doubts whether such an inference is necessary. Indeed, if we eliminate the coefficient $\lambda$ from Dirac's equations, we obtain the following system of equations :

$$
\begin{gathered}
f_{\mu \nu}=A_{\mu, \nu}-A_{\nu, \mu} \\
f \rho^{\nu}, \nu \\
\left\{\delta_{\rho}{ }^{\mu}-\frac{A_{\rho} A^{\mu}}{k^{2}}\right\}=0 .
\end{gathered}
$$

Considering (2) from the algebraic point of view, we see that two cases must be considered :

$$
\text { Case (1) : } \operatorname{det}\left|\delta_{\rho}^{\mu}-\frac{A_{\rho} A^{\mu}}{k^{2}}\right| \neq 0 \text {. }
$$

In this case, equations (2) lead to $f \rho^{\nu}, v=0$, that is, to Maxwell's equations if charged particles are absent.

$$
\text { Case (2) : } \operatorname{det}\left|\delta_{\rho^{\mu}}-\frac{A_{\rho} A^{\mu}}{k^{2}}\right|=0 .
$$

Equation (4) means precisely $A_{\mu} A^{\mu}=k^{2}$. This condition must be fulfilled only if we are looking for a solution for which

$$
k^{2} \lambda=A_{\rho} f^{v}{ }_{, \nu} \neq 0,
$$

that is, if charges are present. Thus (4) need not be fulfilled for a vacuum, or for any regions for which

$$
k^{2} \lambda=A_{\rho} f^{{ }^{v}, \nu}=0 \text {. }
$$

As to Dirac's formulation, the replacement of the equation

$$
\text { by } \quad \begin{aligned}
A_{\mu} A^{\mu}-k^{2} & =0 \\
\lambda\left(A_{\mu} A^{u}-k^{2}\right) & =0
\end{aligned}
$$

leaves all physical conclusions of his electrodynamics unchanged and seems to remove the necessity of reviving the wther concept.

Institute of Theoretical Physics,

L. INFELD

University of Warsaw.

Jan. 31.

INFELD has shown how the field equations of my new electrodynamics can be written so as not to require an æether. This is not sufficient to make a complete dynamical theory. It is necessary to set up an action principle and to get a Hamiltonian formulation of the equations suitable for quantization purposes, and for this the æther velocity is required.

The existence of an æther has not been proved, of course, because my new electrodynamics has not yet justified itself. It will probably have to be modified by the introduction of spin variables before a satisfactory quantum theory of electrons can be obtained from it, and only after this has been accomplished will one be able to give a definite answer to the æther question.

\section{St. John's College, \\ Cambridge.}

P. A. M. DiraC

Feb. 16.

\section{Thermionic Constants of Graphite}

Some years ago, one of us (K. S. K.) suggested a new method of determining the thermionic constants of solids. The method is based on the determination of the saturation vapour pressure of the electron gas in equilibrium with the substance at different known temperatures. In practice, this is done by finding the rate of effusion into vacuum of electrons out of a small hole in a thin wall of a chamber scooped out of the substance. Using a well-known thermodynamic relation, analogous to the ClapeyronClausius equation connecting the temperature variation of the saturation vapour pressure of a given substance with its latent heat of evaporation, one obtains readily the thermionic constants. The work function of graphite was determined by this method by Dr. A. S. Bhatnagar ${ }^{1}$, and of a few other substances by Dr. S. B. L. Mathur ${ }^{2}$; but the $A$ coefficients in Richardson's equation were not determined by these authors, owing to uncertainties in some of the absolute measurements.

We have now improved the experimental technique, particularly to keep the temperature of the chamber uniform throughout, and the apparatus is now enclosed in a sealed glass vessel; this enables us to work in a better vacuum, and to ensure a mean free path of the electrons much larger than the dimensions of the apparatus. In order to eliminate the electrons emitted by the graphite surface adjoining the effusion hole, the surface is suitably covered by a thin mica sheet; a hole punctured in the sheet serves as the effective effusion hole. The area of the effusion hole is determined indirectly from the observed rate of loss of a suitably selected substance like naphthalene, or $p$-dichlorobenzene, kept inside the chamber, in vacuum, the loss being due to the sublimation of

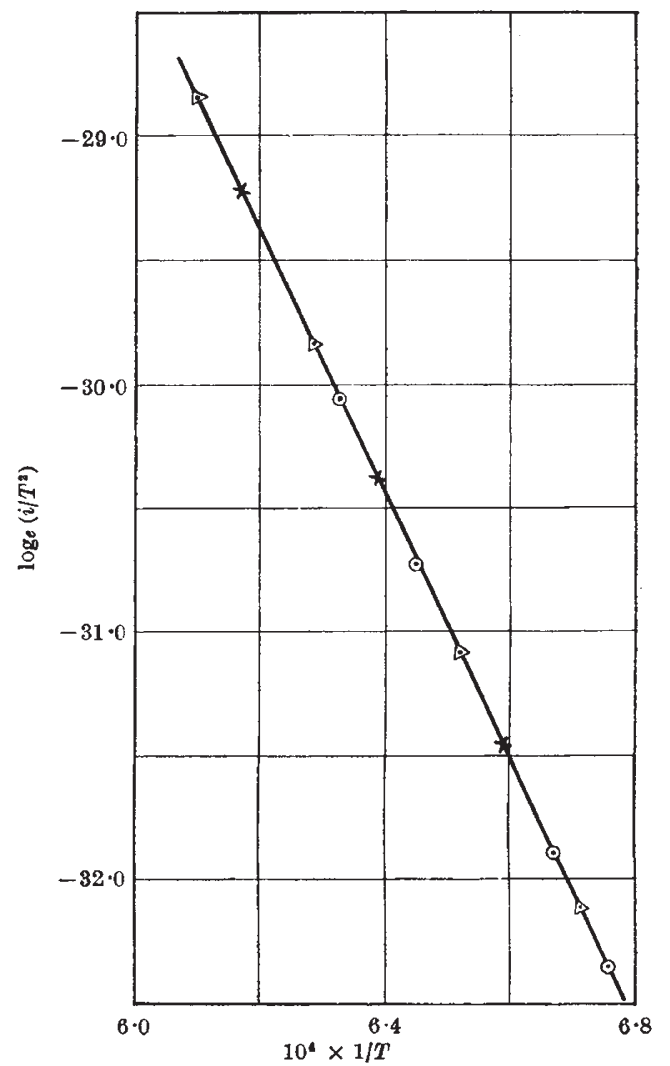

